\title{
TOWARD MORE REALISTIC SIMULATION AND PREDICTION OF DUST STORMS ON MARS
}

\author{
For submission to the \\ Decadal Survey on Planetary Science and Astrobiology \\ Co-Chairs Robin Canup and Phil Christensen
}

\begin{abstract}
Written by:
Claire E. Newman (Aeolis Res.) Tanguy Bertrand (NASA Ames) Joseph Battalio (Yale Univ.)

Mackenzie Day (UCLA)

Manuel de la Torre Juárez (JPL) Meredith K. Elrod (NASA GSFC)

Francesca Esposito (INAF-OAC) Lori Fenton (SETI Inst.) Claus Gebhardt (UAEU)

Steven J. Greybush (Penn. State) Scott D. Guzewich (NASA GSFC) Henrik Kahanpää (FMI)

Melinda Kahre (NASA Ames) Özgür Karatekin (Royal Obs. Belg.) Brian Jackson (Boise State Univ.)

Mathieu Lapotre (Stanford Univ.) Christopher Lee (Aeolis Res.) Stephen R. Lewis (Open Univ.)

Ralph D. Lorenz (APL) Germán Martínez Martínez (LPI) Javier Martin-Torres (Aberdeen U.)

Michael A. Mischna (JPL) Luca Montabone (SSI) Lynn Neakrase (New Mexico State)

Alexey Pankine (SSI) Jorge Pla-Garcia (CAB/SwRI/SSI) Peter L. Read (Univ. of Oxford)

Isaac B. Smith (PSI/York Univ.) Michael D. Smith (NASA GSFC) Alejandro Soto (SwRI)

Aymeric Spiga (Sorbonne Univ.) Christy Swann (NRL SSC) Leslie Tamppari (JPL)

Orkun Temel (Royal Obs. Belgium) Daniel Viudez Moreiras (CAB) Danika Wellington (Ariz. State)

Paulina Wolkenberg (INAF) Gerhard Wurm (Duisburg-Essen) María-Paz Zorzano (CAB)

Contact: Claire E. Newman, Aeolis Research, claire@ aeolisresearch.com, (626) 344-0593
\end{abstract}

Endorsed by:

Ignacio Arruego (INTA)

Jonathan Bapst (JPL)

Edwin Kite (Univ. Chicago)

John E. Moores (York Univ.)

Paula González Pizarro (UCN)

Simone Silvestro (INAF OACN)
Mariah Baker (Smithsonian NASM) Donald P. Banfield (Cornell Univ.)

Matthew Chojnacki (PSI)

Ingrid Daubar (Brown Univ.)

Kevin Lewis ( $\mathrm{JHU})$

John McClean (MIT Haystack Obs.)

Lori Neary (IASB-BIRA)

Manish Patel (Open Univ.)

Scot Rafkin (SwRI)

Mark I. Richardson (Aeolis Res.)

Roland Young (NSSTC/UAEU) 


\section{Toward more realistic simulation and prediction of dust storms on Mars}

Atmospheric dust is a more extreme modifier of weather and climate on Mars than water vapor is on Earth. Global dust storms enshroud Mars in a veil of dust for months and have major implications for past and present climate, geologic history, habitability, and exploration. Yet their mysterious origins mean we remain unable to realistically simulate or predict them.

In this White Paper, we find that key Knowledge Gaps are: A. how dust is lifted; B. constraints on near-surface winds and boundary-layer processes; $\mathbf{C}$. the distribution of mobile surface dust; and $\mathbf{D}$. the key processes and feedbacks by which dust storms begin and evolve.

To make progress in the next decade, we make four Recommendations in order of priority: \#1. Properly accommodate a minimum payload of meteorological and aeolian sensors on future Mars surface missions; \#2. Continue orbital monitoring of the evolving surface dust distribution; \#3. Expand orbital measurements to include winds and full diurnal coverage; and \#4. Continue orbital monitoring and add surface measurements of aerosols during dust storms.

\section{Introduction.}

Major (regional and global) dust storms dominate weather and climate variability on presentday Mars[1,2,3]. Absorption and scattering of visible and IR radiation by dust strongly affect the thermal state of the thin Mars atmosphere[4], while dust provides condensation nuclei for water and $\mathrm{CO}_{2}$ cloud particles, which also affect radiative fluxes [5]. Global dust storms (GDS) occur $\sim$ three times per Mars decade and to date have been observed in only northern fall and winter, when the global circulation is strongest[1]. Tenfold increases in column dust opacity are typical during GDS, with intense vertical motions transporting dust to far higher altitudes than usual[6].

The key processes and feedbacks that produce GDS remain poorly understood, hence current atmospheric models fail to self-consistently simulate observed dust storm activity. This means we have no predictive capability for when GDS may occur, either now or in Mars's past. And crucially, due to the huge impact of GDS on the atmosphere and surface, our lack of ability to simulate realistic dust storms has major implications for Mars science and exploration: from understanding the present climate (\$2.1), to modeling past climates and water cycles $(\$ 2.2)$, to exploring how wind, water, and dust cycles varied over time to interpret geology and assess potential habitability ( $\$ 2.3)$, to quantifying and mitigating risks posed to Mars missions ( $\$ 2.4)$. 2. Impact of better dust storm simulation and prediction on Mars science and exploration.

Here we explain why understanding dust storms is critical to Mars science and exploration. These arguments tie closely to Goals and Objectives identified by the Mars Exploration Program Analysis Group (MEPAG) in their 2020 Goals Document[7], as indicated in each section.

2.1 Dust storms and the present climate of Mars (ties to MEPAG Goal II Objective A).

Global storms produce tens of $\mathrm{K}$ increases in middle-atmosphere temperatures[8], nighttime warming and daytime cooling at and near the surface[2], plus greatly altered wave activity and a stronger global circulation[10]. This in turn affects $\mathrm{CO}_{2}$ cap retreat[11], raises cloud heights[12], and reduces water transport to the South pole[13]. Global storms rearrange surface dust, altering patterns of surface heating. They also strongly enhance interactions between the lower and upper atmosphere: modify thermospheric temperatures and densities[14], increase ionospheric altitudes and photochemical loss rates[15], and raise more water to high altitudes $=>$ more hydrogen loss to space[16]. Hence understanding modern climate processes requires an understanding of GDS.

\subsection{Dust storms and past climates of Mars (ties to MEPAG Goal II Objectives B \& C).}

We must understand the processes and feedbacks that produce today's dust cycle and climate before we can confidently simulate the climates of the past. For example, recent climates were likely modulated mainly by Mars's orbital parameters[17], driving large shifts in annual mean 
pressure as more of the $\mathrm{CO}_{2}$ atmosphere condensed onto, or sublimated from, the surface. Such pressure shifts would have affected wind stress and hence efficiency of dust lifting and intensity of GDS[18], in turn affecting each process described in $\S 2.1$, from cap processes to water escape. 2.3 Dust storms, geology, water, and habitability of Mars (ties to MEPAG Goals I and III).

The strong links between dust, temperature, and the water cycle mean that poor knowledge of past dust cycles greatly increases uncertainty in when and where water ice or liquid may have existed at the surface, especially during the recent past when surface pressures were relatively low and dust dominated heating. The polar layered deposits provide insight into Mars's recent climate history[19] and are hugely affected by dust storm activity, which impacts both polar dust amount \& sedimentation rate[20] and the rate \& nature (snow/ice) of polar volatile deposits[21]. Improved simulation of dust storms would produce more confident predictions of dust and ice accumulation at Mars's surface over orbital cycles, for comparison with observed layering[19]. Correctly modeling the role of storms in recent climates may also be key to understand transient liquid water events, which are of prime interest for Mars. Dust storms also drive surface dust redistribution and strong winds, hence are crucial to landscape evolution[22]. The sedimentary rock record reveals cycles of surface abrasion and dust deposition[23], and wind-driven erosion has removed $\sim 100 \mathrm{~s}$ of $\mathrm{m}$ of material in some areas[24]. Improved storm simulations will result in better modeling of related aeolian activity, clarifying the relative importance of seasonal vs storm winds[22]. Finally, erosion exposes preserved organics not yet degraded by cosmic rays[25] thus the impact of storms on erosion rates aids in assessing the preservation potential of biosignatures. 2.4 Dust storms and Mars exploration (ties to MEPAG Goal IV).

As discussed in \$2.1, major storms strongly perturb upper atmospheric density [14], which is crucial for aerocapture. Even regional storms strongly modify local visibility, temperature, wind, and density in the lower/middle atmosphere, which impact parachutes, navigational imaging, and other EDL processes[26]. Launch windows do not permit spacecraft arriving at or leaving Mars to easily avoid the storm season, so more realistic simulations would allow better risk assessment and mitigation. Long missions will expose astronauts and equipment to a constant barrage of dust[27]. The tiniest grains pose threats to human soft tissues and are harmful to the mechanisms, filters, etc. that are vital to in situ resource utilization[28]. Performance of solar panels, cameras etc. is adversely impacted by dust deposition, which peaks during GDS fallout. Peak insolation may fall to $10 \%$ of normal during GDS[9], so forecasting would be invaluable for solar-powered missions; with more advance warning, Opportunity might have adjusted its tilt and survived[29].

\section{The current state of dust storm simulation and prediction for Mars.}

\subsection{Overview of observed dust storms.}

Only ten GDS have been observed since the first well-observed GDS in 1956 [30]. To date, they have been observed to begin between areocentric solar longitude, Ls 185 and $310^{\circ}$, from a range of precursor storms that often occur in both northern and southern hemispheres, and with specific but widespread primary lifting locations[31]. Dust levels typically return to nominal values 80-100 sols after GDS onset[32]. Regional storms may reach similar peak local opacities but have far lower global mean opacities, cover a far smaller area, and last a few to 20 sols [33].

The 2001 GDS was the first to be observed so early in southern spring (Ls 185 ${ }^{\circ}$, yet in the past two decades such GDS have been most common[32]. In other words, due to the relatively low number of years observed to date, we continue to be surprised by Martian dust storms.

\subsection{The current status of dust storm simulation in Mars climate models.}

We focus here on studies in which simulated storms are determined by the model's dynamics and physics (including parameterized dust processes), as is required for dust storm prediction. 
The main dust processes in a Mars climate model are: surface lifting; atmospheric transport (mixing by unresolved processes and advection by resolved atmospheric winds); scavenging as condensation nuclei for water or $\mathrm{CO}_{2}$ ice; and sedimentation toward the surface[5]. Though other mechanisms may exist[34], climate models typically include lifting by only a) large-scale surface wind stresses above some threshold and b) 'dust devils' (dusty convective vortices)[35]. Once lofted, dust strongly affects atmospheric radiative processes, temperature gradients, and winds, which in turn affect future lifting, while surface dust rearrangement affects surface properties and thus radiative fluxes at the surface. Strong positive feedbacks between dust loading, the circulation, surface wind stress, and lifting are likely crucial to the onset and expansion of major storms [36]. All of these processes and feedbacks must, therefore, be represented correctly to permit the realistic modeling of dust storms - and hence climate and its variability - on Mars.

First-generation models included dust lifting, transport, and sedimentation, and assumed unlimited surface dust availability. High wind stress thresholds were found to produce the best match to the observed large inter-annual variability in GDS, by increasing sensitivity to small year-to-year changes in wind patterns[37]. However, GDS decay rates were far too slow and the range of onset times and spread of onset locations were much smaller than those observed.

Surface dust on Mars is seen to exhaust locally, especially during GDS[38], thus secondgeneration models explored the impact of limited surface dust availability. Dust was found to exhaust in high wind stress areas, with the new primary source regions being those with lower wind stresses and more equal lifting and deposition[39]. Simulations begun from that point, using lower thresholds, had a wider range of onset times and source regions, and more realistic decay rates. However, a feedback between dust cover and thresholds was needed to prevent GDS ceasing as more regions exhausted, and the simulated surface dust distribution was unrealistic. Further, the range of onset times and locations remained too narrow, missing early and late GDS.

The latest models explore coupling between the dust and water cycles, with scavenging and water ice radiative effects included[5]. The ensuing feedbacks sometimes result in greater dust lifting at high northern latitudes around spring equinox and early GDS onset[40]. Yet onset locations, and growth and decay rates, of these early GDS still do not match observations.

In short, while progress has been made, dust storm modeling remains unable to match many aspects of reality. Key Knowledge Gaps that strongly contribute to this are described in $\S 4$. 3.3 The possibility of advance dust storm prediction (forecasting).

As described above, simulations of dust storms do not match the observed range of onset times and locations, especially for early and late GDS. But if models improve to the point that all storm types are represented realistically, will we be able to predict a GDS in advance?

In short-term weather forecasting using Data Assimilation (DA), observed orbital dust and temperature profiles can constrain many aspects of large-scale weather in models[41]. However, constraining the fast-evolving weather features that may be key to dust lifting and transport may require a network of areostationary \&/or polar orbiters, \&/or surface pressure stations $[42,43,44]$. Also, while Mars's atmosphere may be predictable in clear periods, this decreases hugely when the atmosphere is dusty[45]. Overall, for current models and datasets, dust storm prediction via DA seems impossible more than about a sol in advance. Improved atmospheric models and more data impact studies are needed to improve this and to assess true forecasting limits[2,42,44,46].

If surface dust in key regions is found to be a necessary condition for a given storm type to occur, then observed dust cover may provide advance notice that such storms are/are not possible in a given year[39]. Dust source mapping has enabled good progress in dust storm prediction on Earth[47], though such efforts benefit from widespread in situ data on dust cover and processes. 
A proposed coupling between Mars' orbit and rotation, if correct, may dominate inter-annual variability in winds, dust lifting, and hence GDS[30]. To date, simulations show close agreement with the occurrence and seasonal timing of historical GDS in only $~ 60 \%$ of years with good observations, but these statistics may improve as climate models become more sophisticated. 4. Key knowledge gaps for realistic Mars dust storm simulation.

4.1. Knowledge Gap A: How is dust lifted from the surface of Mars?

Lack of in situ data makes it impossible to represent dust lifting correctly in models.

To date, understanding of dust and sand motion on Mars - hence parameterizations of dust lifting and dust fluxes in Mars atmospheric models - has relied on Earth theory[48], computer modeling[49], and wind tunnel experiments at partial Mars conditions[50,51,52,53]. Despite widespread dust lifting observed, expected threshold wind stresses needed to lift dust on Mars were initially far higher than stresses inferred from observations or simulated by models [54]. The proposed explanation was that larger, less-cohesive sand particles are entrained into saltation at lower wind stresses, then fall back to the surface, increase the total stress and cause dust lifting. However the expected saltation threshold was also higher than estimated Mars wind stresses [54].

Several solutions have been proposed in the last decade: saltation bursts due to e.g. strong wind gusts may explain saltation at low overall wind speeds[49,53]; low-cohesion, light-weight dust aggregates, with lower thresholds than individual dust/sand grains, may permit direct dust lifting[51]; low gravity may lower particle cohesion and thus lower thresholds[52]; electrostatics may enhance dust/sand entrainment[55]. This has led to new dust/sand entrainment schemes being proposed for Mars, though the emphasis so far has been on thresholds rather than fluxes.

To date, however, none of these theories or schemes have been verified on Mars itself. Thresholds have been estimated by correlating surface changes with modeled wind stresses [56] or observed wind speeds[57] but not with observed wind stresses. In fact, wind stress has never been measured directly on Mars; wind stress $=u_{*}^{2} \times$ air density, where drag velocity $u_{*}$ depends on both roughness length and atmospheric stability and is not simply a function of wind speed. By definition, $u_{*}$ is the vertical flux of horizontal momentum from the surface, which is given by the covariance of horizontal and vertical wind perturbations, thus a direct measurement requires very fast $(>10 \mathrm{~Hz})$ and accurate 3-D wind measurements, which have never been made on Mars. Drag velocity may be estimated by measuring winds at multiple heights, but even this has only been done once on Mars and was not linked to observed aeolian activity[58]. Similarly, Mars sand fluxes have only been estimated by tracking changes in surface aeolian features[56,57,59] and lack some or all of the coincident data $\left(u_{*}\right.$, grain size, etc.) needed to test theory and improve sand flux parameterizations. Finally, wind-blown dust fluxes have never been measured on Mars.

\subsection{Knowledge Gap B: How good are our predictions of winds and boundary layer processes?} Despite eight successfully-landed Mars missions, we still lack basic meteorological data for validating near-surface winds and boundary layer processes in Mars atmospheric models.

Knowing how dust lifting relates to local conditions (§4.1) is not enough. We must also be able to simulate those local conditions correctly in atmospheric models before we can simulate realistic patterns of dust lifting, and hence dust storm onset and growth. Mixing and advection of lofted dust must also be correct if models are to simulate realistic storms. Unfortunately, current Mars models differ hugely from each other, and from observations, in their predictions of key variables, such as wind stress and boundary layer mixing[60,61]. This is likely a result of poor representation of boundary layer processes in the models. On Earth, in situ data are a prerequisite for developing model boundary layer schemes[62]. These data include turbulent fluxes of heat and momentum at some height (requiring high-accuracy, fast response-time [ $>10 \mathrm{~Hz}] 3-\mathrm{D}$ winds, 
as described in §4.1) and eddy characteristics as a function of height (requiring fast, accurate wind and temperature data at multiple heights). For Mars, such measurements have never been made, hence the data needed to design and test Mars-specific schemes are not yet available[63].

Another major issue is the accommodation of meteorological sensors. A standalone, thin meteorological mast that perturbs the flow very little, set away from artificial sources of heating / cooling, would be ideal. Yet wind sensors are often mounted where flow from several directions is badly perturbed, while air temperature sensors are placed near to RTGs or solar panels[3,9,61].

4.3. Knowledge Gap C: What is the surface distribution of mobile dust on Mars?

A long timeline of orbital data is needed to characterize Mars's surface dust distribution.

Models that allow realistic depletion/exhaustion of surface dust produce better dust storm simulations (§3.2). Such models self-consistently rearrange dust (via modeled lifting/deposition), but knowing the true surface dust distribution is vital, both as an initial state and to assess how well the model evolves it. Determining the distribution of surface dust is not easy, however. Low thermal inertia $=>$ thick dust cover but high values can have many interpretations[64]. And while low albedo $=>$ dust-free areas, high values may mean only microns-deep layers [65] or immobile duststone[66]. IR spectroscopy can give maps of composition, grain size and maybe cementation state[64,67,68], yet without in situ data to constrain them, such maps will be highly unreliable.

For these reasons, the variation with time of surface properties is currently most useful to determine Martian surface dust abundance and mobility. E.g. regions that darken during a GDS were likely exhausted of dust by storm lifting[38]. By accumulating such observations over a large number of major storms - each unique in its combination of onset timing, sources, and intensity - we can build up a picture of dust availability. Also vital are ground truth data on soil properties, and on dust lifting / deposition rates, to enable validation of orbital observations[69].

4.4. Knowledge Gap D: What are the processes and feedbacks behind dust storm evolution? More data on winds and aerosols during storms, at all altitudes and times of sol, are needed to understand the radiative-dynamical-microphysical processes and feedbacks involved.

Positive feedbacks on local winds or the large-scale circulation may increase dust lifting and drive GDS growth[35,70], with significant lifting and vertical mixing likely driven by processes that peak at certain times of sol[6,71]. However, orbital observations are often restricted to a few local times, hence miss major phenomena that vary diurnally. Advection of lofted dust is also vital to storm growth[72] yet direct measurements of Mars winds are only available within $2 \mathrm{~m}$ of the surface at only a handful of locations[3]. Dust-water ice interactions may be key to producing early GDS (\$3.2) but are very sensitive to poorly-known parameters such as nucleation rates and aerosol size distributions[5]. Similarly, storm dust fallout may be hastened by scavenging of dust particles by ice[5,73], while lifting may cease due to source dust depletion[39] or the suppression of certain circulation components (e.g. slope winds) at high opacities[73]. Hence measuring the evolution of atmospheric conditions and aerosol properties over the lifecycle of a range of storm types, from the surface to storm top, is needed to represent these interactions correctly in models.

\section{Recommendations for the coming decade.}

Below we make four recommendations, in order of priority, for steps we can take over the next decade to move toward the realistic simulation and prediction of Martian dust storms.

\section{Recommendation \#1: Include a minimum payload of meteorological and aeolian sensors,} with adequate accommodation, on all future Mars surface missions.

Knowledge Gaps A and B can be filled by performing the following set of meteorological and aeolian measurements simultaneously on the Martian surface:

Wind stress: High-accuracy, fast-response 3-D anemometers are needed to measure $u_{*}$ and 
hence wind stress directly (\$4.1). Such sensors have been developed for Mars over several years, and are now sufficiently low-mass and low-power to permit inclusion on surface missions[74]. Boundary layer: Winds and temperatures at multiple heights, plus vertical fluxes of radiation, heat, and momentum, are key to develop and validate model boundary layer schemes $(\$ 4.2)$. Dust/sand flux \& sizes: These are needed to determine when thresholds are exceeded and to quantify the relationship between dust flux, sand flux, and local conditions. Sand impact and lofted dust abundance sensors will be carried on ExoMars 2022[75], while more sophisticated flux sensors are actively in development and should be available soon in the next decade[76]. Soil properties: Grain size distribution affects thresholds and is key to interpret lofted dust size distributions. Soil moisture affects cohesion, and temperature may also impact dust lifting[34]. Electrostatics: E-field and conductivity are needed to characterize the impact of charging on entrainment thresholds and on dust and sand fluxes[55]. ExoMars 2022 will carry such sensors.

We therefore recommend an aeolian/meteorological Mars surface mission to measure:

* Fast $(>10 \mathrm{~Hz})$, accurate 3-D winds @ 1.5m and 1-D winds @ two other heights $(\sim 0.5,1 \mathrm{~m})$

* Dust and sand fluxes and size distributions * Surface grain size distribution and abundance

* Pressure and surface temperature * Air temperature @ $~ 0.5,1,1.5 \mathrm{~m} *$ Radiative fluxes

* E-field and electrical conductivity * Air and soil water content * Soil temperature

We also recommend that such 3-D wind, pressure, temperature, dust/sand, and radiative measurements be made on all future surface missions. Improved aeolian and meteorological surface measurements are also urged by other White Papers[63,68,77,78,79] and MEPAG[Z].

As discussed in $\$ 4.2$, meteorological sensors have historically had quite poor placement with respect to mechanical \&/or thermal perturbations, rendering some measurements nearly useless.

We thus further recommend that accommodation of meteorological sensors be considered early in mission planning and a standalone meteorological mast be utilized when possible.

Recommendation \#2: Continue orbital monitoring of surface property variations. Knowledge Gap C can only be filled by determining where dust is available to be lifted, which requires long-term monitoring of the temporal evolution of surface properties on Mars.

As noted in $\S 3.3$, such monitoring could potentially let us predict whether a given dust storm can occur in the current Mars year. The problem is that, to date, we have only measured surface changes from a handful of major dust storms on Mars, which have shown great diversity.

We therefore recommend that orbital measurements of global surface changes continue as a priority over the next decade, with new instruments flown to prevent an interruption in coverage of surface properties (and their time variation) from the loss of aging spacecraft. This recommendation aligns with other White papers [68,78] and the NEX-SAG report[80].

Recommendation \#3: Expand orbital measurements to include winds and diurnal coverage. To understand the dynamical feedbacks that control how dust storms evolve (part of Knowledge Gap D), observing the diurnal variation of winds and dust distributions from orbit is vital.

Strong winds trigger storm onset, while dust lifting and transport are key to storm growth. Feedbacks between winds and dust loading may control most aspects of storm evolution. Yet we still lack any direct, global wind observations above $\sim 2 \mathrm{~m}$ altitude, with few measurements below this too. Simultaneous observations of winds and dust distributions from orbit are thus urgently needed, to validate models and determine feedbacks between the circulation and dust storms. Instruments to measure wind from Mars orbit are high-TRL and could fly in the next decade[81].

Many processes - especially dust lifting, vertical mixing, and transport - vary greatly with 
time of sol, hence full diurnal coverage is needed to study them during storms. Areostationary satellites or constellations of areostationary and polar satellites could monitor dust and related meteorology with constant coverage, just as Earth satellites monitor hurricanes[2,43,44].

We recommend that direct, global measurements of atmospheric winds be made from orbit, along with full diurnal sampling of dust distributions, winds, and temperatures. This recommendation aligns with other White Papers[43,82] and the NEX-SAG report[80].

Recommendation \#4: Continue observations of aerosol abundance and size distributions during storms from orbit and make observations from the surface during storms. To understand dust storm radiative and microphysical feedbacks (rest of Knowledge Gap D) we must monitor aerosols from orbit and the surface over more cycles of major dust storms.

The dust and water cycles interact radiatively and microphysically, with onset, growth, and decay of GDS potentially dependent on this to a huge extent. Thus continued aerosol monitoring from orbit across a range of storm types is vital, with surface measurements (e.g. via Lidar) also needed to provide insight into the near-surface changes that are often obscured by dust clouds.

We recommend continued monitoring of aerosol abundance and size distributions from orbit, and new surface measurements of lower atmosphere aerosols during major storms. This recommendation aligns with other White Papers[43,77,79] and the NEX-SAG report[80].

References are provided in full at: http://bit.ly/DustStormsWhitePaperReferences

1) Read et al. RPP 2015. 2) Forget \& Montabone ICES 2017. 3) Martínez et al. SSR 2017. 4)

Wolff et al. Atmos. Clim. Mars 2017. 5) Navarro et al. JGR 2014. 6) Heavens et al. JGR 2019. 7) MEPAG 2020. 8) Kass et al. GRL 2019. 9) Viúdez-Moreiras et al. JGR 2019. 10) Wilson \& Hamilton JAS 1996. 11) Bonev et al. PSS 2008. 12) Stcherbinine et al. JGR 2020. 13) Pankine \& Tamppari Icarus 2019. 14) Chaufray et al. GRL 2020. 15) Fang et al. JGR 2020. 16) Aoki et al. JGR 2019. 17) Laskar et al. Icarus 2004. 18) Haberle et al. Icarus 2005. 19) Becerra et al. GRL 2017. 20) Hvidberg et al. Icarus 2012. 21) Colaprete et al. Nat. 2005. 22) Fenton Caltech thesis 2003. 23) Grotzinger \& Milliken Sed. Geo. Mars 2012. 24) Golombek et al. JGR 2014. 25) Farley et al. Sci. 2014. 26) Chen et al. IEEE ACP 2010. 27) Spry et al. LPSC 2017. 28) McClean PSS 2020. 29) Callas JPL Rep. 2019. 30) Newman et al. Icarus 2019. 31) Wang \& Richardson Icarus 2015. 32) Wolkenberg et al. JGR 2020. 33) Cantor et al. JGR 2001. 34) Neakrase et al. SSR 2016. 35) Newman et al. JGR 2002. 36) Kahre et al. Atmos. Clim. Mars 2017. 37) Basu et al. JGR 2006. 38) Szwast et al. JGR 2006. 39) Newman \& Richardson, Icarus 2015. 40) Kahre et al. MAMO 2011. 41) Lewis et al. Icarus 2007. 42) Lewis et al. PSS 1996. 43) Montabone et al. W. Paper 2020. 44) Gebhardt \& Abuelgasim PEPS 2019. 45) Newman et al. QJRMS 2004. 46) Navarro et al. ESS 2017. 47) Shao et al. AR 2011. 48) Kok et al. RPP 2012. 49) Sullivan \& Kok JGR 2017. 50) Greeley et al. GRL 1980. 51) Merrison et al. Icarus 2007. 52) Musiolik et al. Icarus 2018. 53) Swann et al. GRL 2020. 54) Pollack et al. Icarus 1976. 55) Esposito et al. GRL 2016. 56) Ayoub et al. Nat. Comm. 2014. 57) Bridges et al. JGR 2017. 58) Sullivan et al. JGR 2000. 59) Bridges et al. Nat. 2012. 60) Newman et al. SSR 2020. 61) Newman et al. Icarus 2017. 62) Huang et al. BLM 2017. 63) Rafkin et al. W. Paper 2009. 64) Ruff \& Christensen JGR 2002. 65) Wells et al. Icarus 1984. 66) Bridges \& Muhs Sed. Geo. Mars 2012. 67) Yin et al. AE 2007. 68) Titus et al. W. Paper 2020. 69) Kinch et al. ESS 2015. 70) Bertrand et al. JGR 2020. 71) Rafkin et al. Nat. 2002. 72) Gillespie et al. JGR 2020. 73) Pollack et al. JGR 1979. 74) Banfield et al. JASA 2016. 75) Scaccabarozzi et al. Measurement 2018. 76) Merrison et al. PSS 2006. 77) Diniega et al., W. Paper 2020. 78) Dundas et al. W. Paper 2020. 79) Spry et al. W. Paper 2020. 80) NEX-SAG 2015. 81) Read et al. PSS 2018. 82) Guzewich et al. W. Paper 2020. 